\title{
Bristol Stool Type 3
}

National Cancer Institute

\section{Source}

National Cancer Institute. Bristol Stool Type 3. NCI Thesaurus. Code C125396.

A sausage shape with cracks in the surface; normal stool. 